\title{
THE USE OF TRIBROMOISOCYANURIC ACID TO DISTINGUISH AMONG PRIMARY, SECONDARY AND TERTIARY ALCOHOLS
}

\author{
Lívia T. C. Crespo* \\ Departamento de Química Orgânica, Instituto de Química, Universidade Federal do Rio de Janeiro, CP 68545, 21945-970 Rio de \\ Janeiro - RJ / Instituto Federal de Educação, Ciência e Tecnologia do Rio de Janeiro, campus Duque de Caxias, Av. República do \\ Paraguai, 120, 25050-100 Duque de Caxias - RJ, Brasil \\ Marcio C. S. de Mattos e Pierre M. Esteves \\ Departamento de Química Orgânica, Instituto de Química, Universidade Federal do Rio de Janeiro, CP 68545, 21945-970 Rio \\ de Janeiro - RJ, Brasil \\ Recebido em 2/4/12; aceito em 17/8/12; publicado na web em 1/2/2013

\begin{abstract}
Primary, secondary and tertiary alcohols can be easily distinguished due to their reactivity towards tribromoisocyanuric acid (TBCA). The test is performed by adding TBCA to the alcohol in a test tube heated in a boiling water bath. Orange color develops in the tube containing the primary alcohol, light yellow is observed in the tube containing the secondary alcohol while the tertiary alcohol results in a colorless mixture.
\end{abstract}

Keywords: tribromoisocyanuric acid; alcohols; spot test.

\section{INTRODUCTION}

Although spectroscopic methods are capable of differentiating primary, secondary and tertiary alcohols, the use of chemical methods for this purpose is still of great value, as these techniques are cheaper and of easier access to organic chemists. Among the chemical tests used in the differentiation of alcohols, the Lucas, ${ }^{1,2}$ chromic acid, ${ }^{1,2}$ xanthate $^{3,4}$ and vanadium oxinate ${ }^{4}$ tests are the most employed for this purpose. However, some disadvantages, such as the toxic compounds involved, e.g. $\mathrm{Cr}(\mathrm{VI})$ salts in the chromic acid test and carbon disulfide in the xanthate test, and the need for more than one test for the differentiation of the three classes of alcohols, as shown in Figure 1, prompts the search for new alternatives.

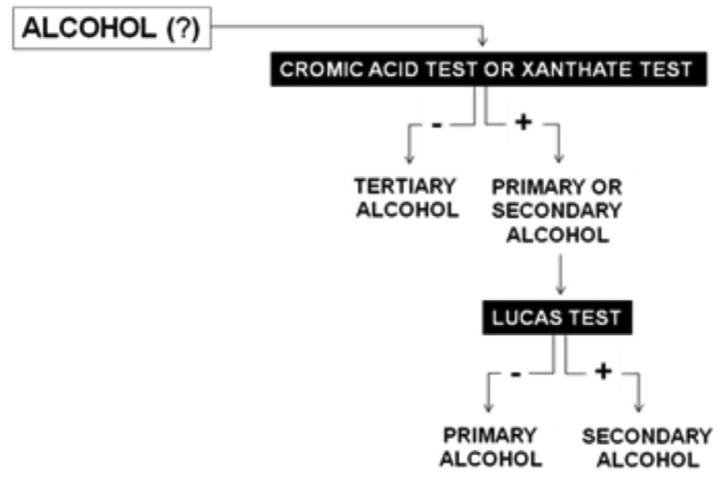

Figure 1. Testing scheme for the differentiation of alcohols

In 1997, Hiegel and Chaharmohal developed a new method for the differentiation of primary and secondary alcohols based on the rate of oxidation of trichloroisocyanuric acid (TCCA, Figure 2) and formation of a precipitate of cyanuric acid. ${ }^{5,6}$ According to the authors, secondary alcohols react faster than primary alcohols with TCCA in acid solution of acetonitrile.

In this study, a spot test for distinguishing alcohols was developed

*e-mail: livia.vilela@ifrj.edu.br<smiles>O=c1n(Cl)c(=O)n(Cl)c(=O)n1Cl</smiles><smiles>O=c1n(Br)c(=O)n(Br)c(=O)n1Br</smiles>

Figure 2. Structures of trichloroisocyanuric (TCCA) and tribromoisocyanuric (TBCA) acids

based on the oxidation reaction of alcohols by tribromoisocyanuric acid (TBCA, Figure 2). This reagent is an easily handled white solid that can be safely prepared from isocyanuric acid. ${ }^{7}$

\section{RESULTS AND DISCUSSION}

The test to distinguish the alcohols was performed in 3 different test tubes containing about 8 drops of a primary (butanol), a secondary (sec-butanol) and a tertiary (tert-butanol) alcohol, respectively. Approximately $20 \mathrm{mg}$ of TBCA was added to each tube containing the alcohols and after 2 min in a boiling water bath an orange color developed in the tube containing the primary alcohol, light yellow color in the tube containing the secondary alcohol while a colorless liquid was observed in the tube containing the tertiary alcohol (Figure 3 ). It was found that the test requires the use of excess alcohol to perform the differentiation among the different colors.

A more detailed analysis of the experiment shows that when a secondary alcohol is added to a test tube containing TBCA the mixture turned to yellow almost immediately. The color intensity increases rapidly until, after a few seconds (when the mixture is heated in a boiling water bath) the mixture loses color and became light yellow. On the other hand, in the test tube containing a primary alcohol the mixture remains unchanged for a few seconds, slowly turning yellow and then orange, remaining this color for several days.

In order to determine the influence of solvent on the test, 8 drops of alcohol were diluted in $1 \mathrm{~mL}$ of several solvents and $20 \mathrm{mg}$ of TBCA were added to this mixture (Table 1). Alkanes, esters, nitriles, $\mathrm{CH}_{2} \mathrm{Cl}_{2}$ and benzene were found not to interfere in the test. However, 

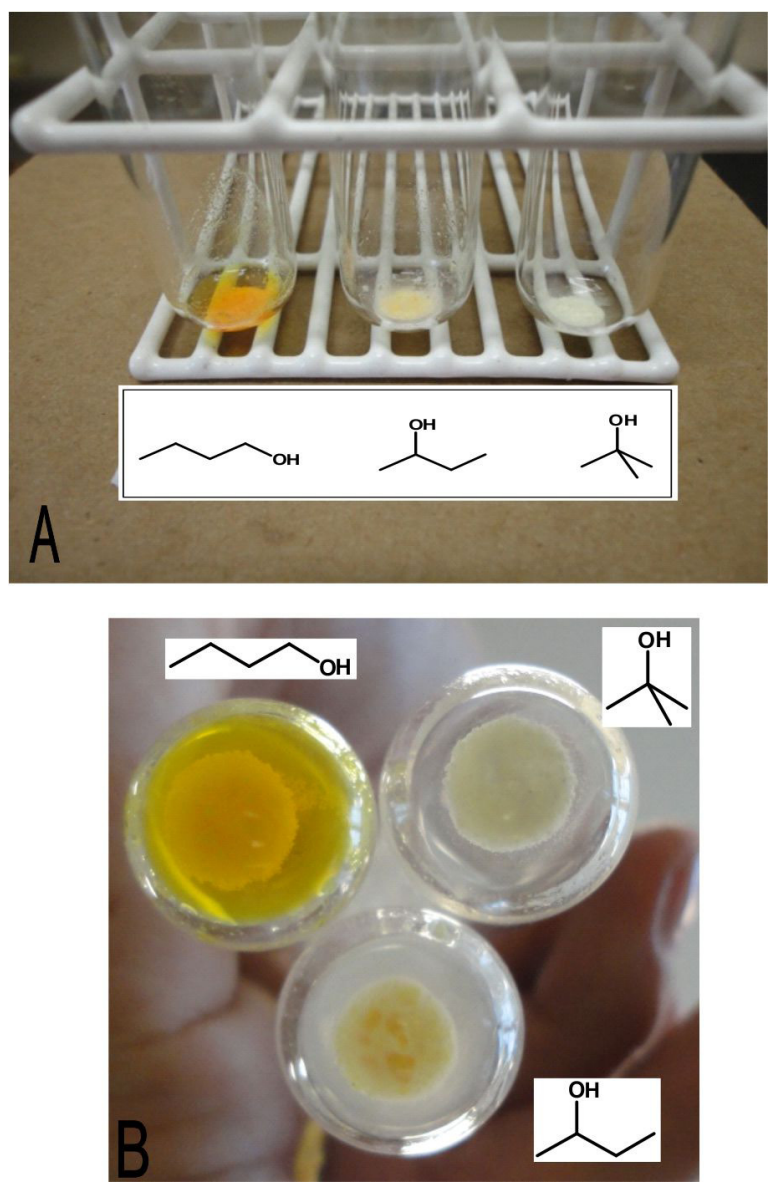

Figure 3. Spot test of TBCA with butanol, sec-butanol and tert-butanol

the presence of the amine group interfered in the result (turbidity). This is somewhat expected, since electron-rich groups such as amines and thiols, can reduce almost instantaneously both TBCA and TCCA exothermically, affording isocyanuric acid (responsible for the turbidity) in an exothermic reaction, which cause some smoking/ gas evolution in the test tube. These effects can therefore interfere in the tests. Ethers and ketones in excess $(1 \mathrm{~mL})$ also interfere with the test. However, smaller proportions of these functions may be present without generating false positive results.

Based on the above results, the spot test was extended to other alcohols (Table 2 and Figure 1S, supplementary material) obtaining results consistent with the previous ones.

The utility of the test for solid alcohols was evaluated by solubilizing them in $1 \mathrm{~mL}$ of hexane. The solution was then poured into the test tube containing $20 \mathrm{mg}$ of TBCA. In this case, the color development was found to be slow, requiring $20 \mathrm{~min}$ for completion. Furthermore, in the case of secondary alcohols, the test initially resulted in a yellow color in the same manner as the secondary liquid alcohols, but in the end the liquid became colorless. This phenomenon is probably due to long heating time.

Unsaturated alcohols (Figure 4) led to ambiguous results because there was no change in color of the liquid, which behaved similarly to tertiary alcohols. Thus, the TBCA cannot be employed for these cases. This result is possibly due to the reaction of TBCA with the unsaturated function groups (alkenes, alkynes, etc.) leading to bromine consumption.<smiles>C=CCO</smiles><smiles>OC/C=C/c1ccccc1</smiles><smiles>CC1=CC[C@@H](C(C)(C)C)C[C@H]1O</smiles><smiles>CC1=CCC(C(C)(C)O)CC1</smiles>

Figure 4. Unsaturated alcohols tested

It was observed by following the reaction using ${ }^{1} \mathrm{H},{ }^{13} \mathrm{C} \mathrm{NMR}$ and GC-MS that butanol, in the presence of TBCA, generates butyl butyrate as the major product. The analysis of the reaction by UV-Vis showed that the staining observed was due to the formation of $\mathrm{Br}_{2}$ in the reaction system. To confirm this, the reaction of butanol with TBCA in a water bath was repeated and, after 2 min, cyclohexene was added to the reaction medium and an immediate bleaching of the mixture was observed. Analysis by GC-MS revealed the presence of 1,2-dibromocyclohexane among the products, confirming the in situ formation of $\mathrm{Br}_{2}$.

A similar study using sec-butanol with TBCA showed the formation of 2-butanone as the main product. By monitoring the reaction by $\mathrm{UV}-\mathrm{Vis}$ it was found that the coloration was again due to formation of $\mathrm{Br}_{2}$ and it was observed that bleaching occurs for the formation of a-bromo-butanone, as disclosed by GC-MS.

The results suggest that the primary alcohol is oxidized to its respective aldehyde and ester with concomitant $\mathrm{Br}_{2}$ formation, which produces orange staining in the reaction medium (Scheme 1).

Table 1. Spot test of differentiation of alcohols diluted in different solvents (1 mL)

\begin{tabular}{|c|c|c|c|c|}
\hline Solvent & $\mathrm{t}(\mathrm{min})$ & & $\mathrm{OH}$ & ${ }^{\mathrm{OH}}$ \\
\hline hexane & 5 & orange & light yellow & colorless \\
\hline ethyl acetate & 15 & dark yellow & light yellow & colorless \\
\hline acetonitrile & 10 & dark yellow & light yellow & colorless \\
\hline methylene dichloride & 10 & orange & light yellow & colorless \\
\hline butylamine & 10 & cloudy & cloudy & cloudy \\
\hline butylamine * & 10 & cloudy & cloudy & cloudy \\
\hline ethyl ether & 10 & orange & light yellow & light yellow \\
\hline ethyl ether * & 10 & dark yellow & light yellow & colorless \\
\hline acetone & 2 & colorless & colorless & colorless \\
\hline acetone* & 5 & dark yellow & light yellow & colorless \\
\hline
\end{tabular}

*8 drops 
Table 2. Results of the spot test of alcohols with TBCA
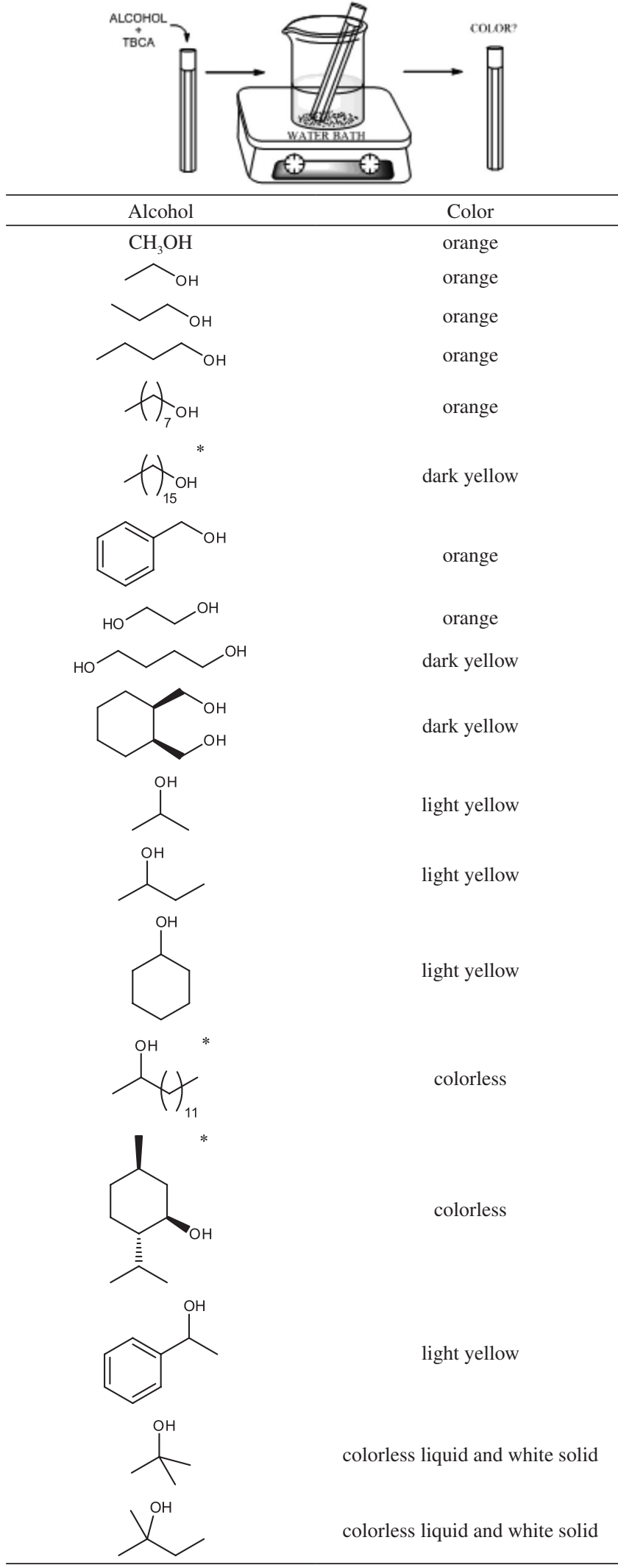

*1.3 mmol of alcohol dissolved in $1 \mathrm{~mL}$ of hexane

On the other hand, when the alcohol is secondary, oxidation takes place with the formation of ketone and $\mathrm{Br}_{2}$ (indicated by the color orange) which react together rapidly to form the corresponding

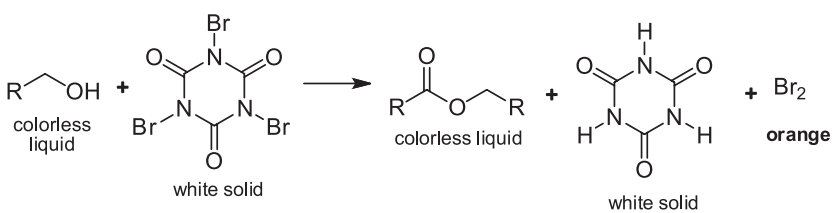

Scheme 1. Reaction of oxidation of a primary alcohol with TBCA

$\alpha$-bromo-ketone, the reaction leading to a light yellow or colorless mixture (depending on whether consumption of $\mathrm{Br}_{2}$ was partial or total) (Scheme 2)

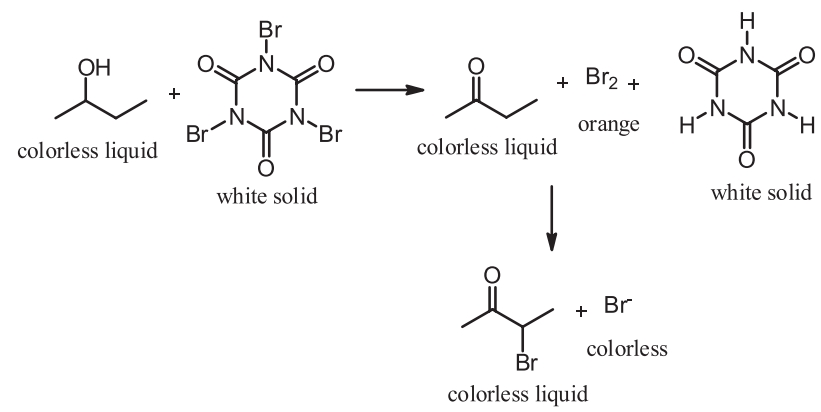

Scheme 2. Reaction of oxidation of a secondary alcohol with TBCA

\section{CONCLUSION}

The use TBCA in the differentiation of primary, secondary and tertiary alcohols proved to be a straight forward and practical test for laboratory use. The different colors are due to the different reactivities of the alcohols with TBCA. TBCA in the presence of a primary alcohol is oxidized to an ester whilst $\mathrm{Br}_{2}$ is formed as a coproduct turning the reaction mixture orange. Secondary alcohols are oxidized to their corresponding ketones, again generating bromine as a co-product. However, the generated ketone further reacts with $\mathrm{Br}_{2}$ affording a-bromoketones, leading to the bleaching reaction medium. By contrast, tertiary alcohols cannot be oxidized by TBCA, therefore no color change is observed with these compounds.

\section{EXPERIMENTAL}

In a test tube containing about $20 \mathrm{mg}$ of tribromoisocyanuric acid (TBCA), 8 drops of the alcohol were added (solid alcohols were dissolved in hexane). The test tube was left in a boiling water bath for 2 min. The tubes containing the primary alcohol turned orange, those containing secondary alcohols exhibited a light yellow color whereas no change in color was observed in the tubes containing tertiary alcohols.

\section{SUPPLEMENTARY MATERIAL}

Photos of the color test for distinguishing primary, secondary and tertiary alcohols are available at http://quimicanova.sbq.org.br, in pdf file with free access.

\section{ACKNOWLEDGMENTS}

We thank CNPq and FAPERJ for financial support.

\section{REFERENCES}

1. Pavia, D. L.; Lampman, G. M.; Kriz, G. S.; Engel, R. G.; Introduction to Organic Laboratory Techniques: A Small-Scale Approach, $2^{\text {nd }}$ ed., Brooks Cole: Pacific Grove, 2005. 
2. Shriner, R. L.; Fuson, R. C.; Curtin, D. Y.; Morrill, T. C.; The Systematic Identification of Organic Compounds, $6^{\text {th }}$ ed., Jonh Wiley \& Sons: New York, 1980.

3. Costa Neto, C.; Análise Orgânica. Métodos e Procedimentos para caracterização de Organoquímios, UFRJ: Rio de Janeiro, 2004, vol. 1, p. 493-495.
4. Fiegl, F.; Anger, V.; Spot Tests in Organic Analysis, $7^{\text {th }}$ ed., Elsevier Scientific Publishing Company: New York, 1966.

5. Hiegel, G. A.; Chaharmohal, A. K.; J. Chem. Educ. 1997, 74, 423.

6. Tilstam, U.; Weinmann, H.; Org. Proc. Res. Develop. 2002, 6, 384.

7. De Almeida, L. S.; Esteves, P. M.; de Mattos, M. C. S.; Synlett 2006 1515. 


\section{THE USE OF TRIBROMOISOCYANURIC ACID TO DISTINGUISH AMONG PRIMARY, SECONDARY AND TERTIARY ALCOHOLS}

\section{Lívia T. C. Crespo*}

Departamento de Química Orgânica, Instituto de Química, Universidade Federal do Rio de Janeiro, CP 68545, $21945-970$ Rio de Janeiro - RJ / Centro Federal de Educação, Ciência e Tecnologia do Rio de Janeiro, campus Duque de Caxias, Av. República do Paraguai, 120, 25050-100 Duque de Caxias - RJ, Brasil

Marcio C. S. de Mattos e Pierre M. Esteves

Departamento de Química Orgânica, Instituto de Química, Universidade Federal do Rio de Janeiro, CP 68545, $21945-970$ Rio de Janeiro - RJ, Brasil
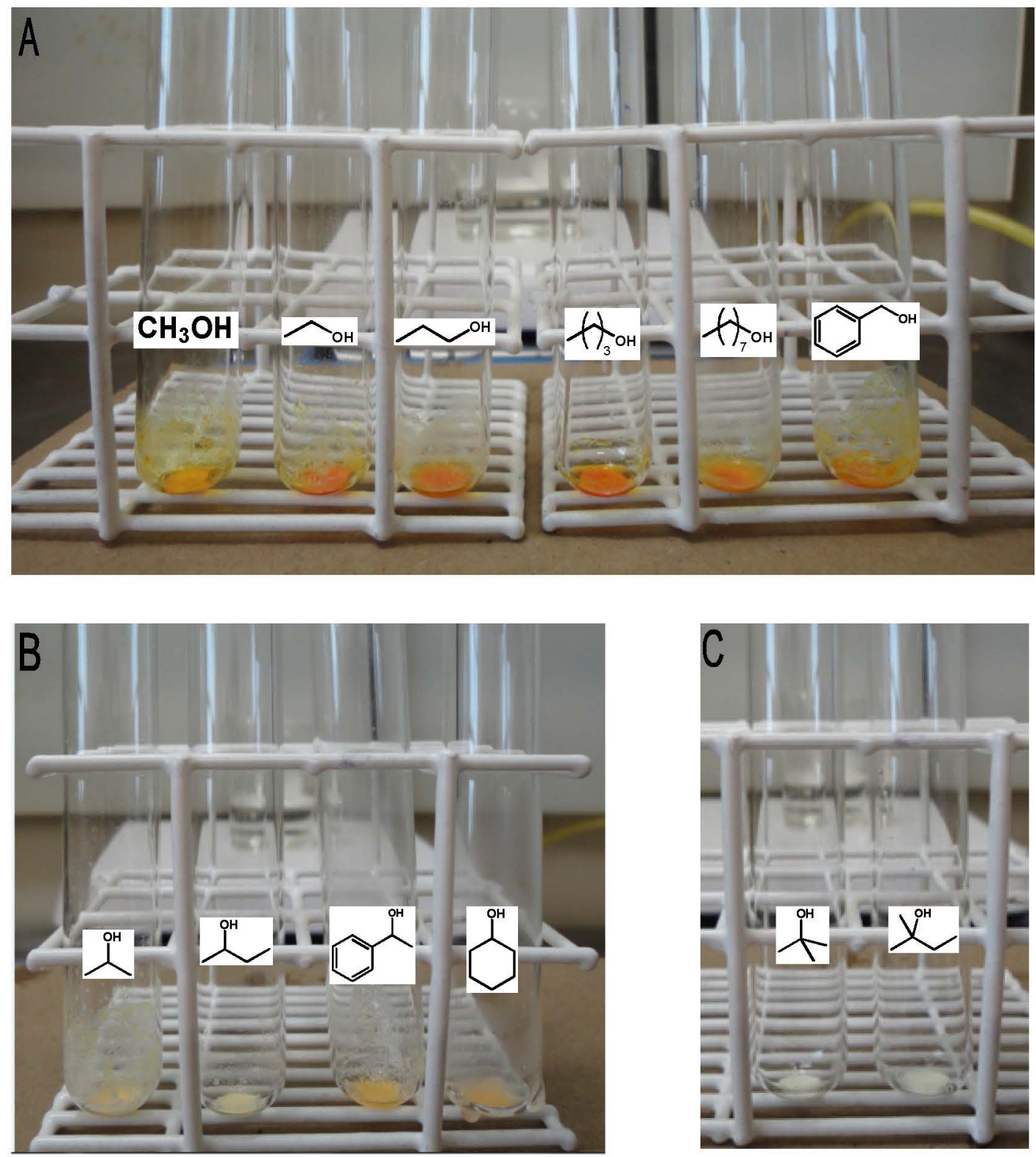

Figure 1S. Spot test of TBCA with (A) primary alcohols; (B) secondary alcohols; $(C)$ tertiary alcohols 